EINDHOVEN UNIVERSITY OF TECHNOLOGY

Department of Mathematics and Computing Science

Memorandum COSOR 89-15

The output-stabilizable subspace

and linear optimal control

A.H.W. Geerts and M.L.J. Hautus

Eindhoven University of Technology

Department of Mathematics and Computing Science

P.O. Box 513

$5600 \mathrm{MB}$ Eindhoven

The Netherlands

Eindhoven, June 1989

The Netherlands 


\title{
The output-stabilizable subspace and linear optimal control
}

\author{
A.H.W. Geerts \& M.L.J. Hautus
}

\section{Abstract}

Properties of a certain subspace are linked to well-known problems in system theory.

\section{Keywords}

Output stabilizability, linear-quadratic problem, singular controls, structure algorithm, dissipation inequality.

\section{Introduction}

Consider the following finite-dimensional linear time-invariant system $\Sigma$ :

$$
\begin{aligned}
& \dot{x}(t)=A x(t)+B u(t), x(0)=x_{0}, \\
& y(t)=C x(t)+D u(t),
\end{aligned}
$$

where for all $t \geq 0, x(t) \in \mathbb{R}^{n}, u(t) \in \mathbb{R}^{m}$ and $y(t) \in \mathbb{R}^{r}$, and the input $u(\cdot)$ is required to be an element of

$$
C_{s m}^{m}:=\left\{u:[0, \infty) \mapsto \mathbb{R}^{m} \mid \exists_{\varepsilon>0} \exists_{v \in C^{\infty}\left((-\varepsilon, \infty) \mapsto \mathbb{R}^{m}\right)} \forall_{l \geq 0}: u(t)=v(t)\right\},
$$

the class of smooth controls. Moreover, without loss of generality, we may assume that $\left[B^{\prime} D^{\prime}\right]^{\prime}$, $[C D]$ is injective and surjective, respectively.

For the case $D=0$, we now recall Wonham's Output Stabilization Problem ([11, Section 4.4]):

(OSP): Given the system $\Sigma$ with $D=0$. Find a feedback map $F: \mathbb{R}^{m} \mapsto \mathbb{R}^{n}$ such that with the input $u=F x$, we have $y(t) \rightarrow 0$ for any initial value $x_{0}$.

If this problem has a solution, then $\Sigma$ (with $D=0$ ) is called output stabilizable. A necessary and sufficient condition for the output stabilizability was provided by [11, Theorem 4.4]. A slightly different formulation of the condition was given in [5, Theorem 4.10], where it was shown that OSP has a solution if and only if $\mathbb{R}^{n}=\mathbf{S}^{-}$, where the subspace $\mathbf{S}^{-}$was defined in terms of $(\xi, \omega)$-representations. More generally, this subspace also plays a role in the outputstabilization problem under disturbances, i.e., the problem of achieving BIBO stability in the presence of a disturbance input term Eq. Then, it turns out, the condition is: $\operatorname{im}(E) \subset \mathbf{S}^{-}$. 
Next, let

$$
J\left(x_{0}, u\right):=\int_{0}^{\infty} y^{\prime}\left(x_{0}, u\right) y\left(x_{0}, u\right) d t,
$$

with $y\left(x_{0}, u\right)=C x\left(x_{0}, u\right)+D u$ (compare (1.1b)), and $x\left(x_{0}, u\right)$ denotes the solution of (1.1a) for given $x_{0}$ and $u \in C_{s m}^{m}$. We introduce the Linear-Quadratic optimal Control Problem:

(LQCP): for all $x_{0}$, determine $J^{-}\left(x_{0}\right):=\inf \left\{J\left(x_{0}, u\right) \mid u \in C_{s m}^{m}\right\}$ and, if for all $x_{0} \in \mathbb{R}^{n}$, $J^{-}\left(x_{0}\right)<\infty$, then compute, if one exists, all optimal controls (i.e. all controls $u^{*} \in C_{s m}^{m}$ such that $\left.J^{-}\left(x_{0}\right)=J\left(x_{0}, u^{*}\right)\right)$.

We will call LQCP solvable if for all $x_{0}, J^{-}\left(x_{0}\right)<\infty$ and if for every $x_{0}$ there exists an optimal input $u^{*}$ (i.e. an input $u^{*}$ such that $J^{-}\left(x_{0}\right)=J\left(x_{0}, u^{*}\right)$ ). In this paper we shall see that the subspace $S^{-}$is relevant for the issue of LQCP-solvability.

The above-mentioned problem is called regular if $\operatorname{ker}(D)=0$ and singular if $\operatorname{ker}(D) \neq 0$. The regular case is well established and considered classical. Curiously, the problem of finding necessary and sufficient conditions for solvability of the problem has found little attention, even in the regular case. Usually, one is satisfied with the statement that the problem is solvable if $(A, B)$ is stabilizable (see e.g. [10, Propositions 9-10]). Of course, this condition is not necessary (if $C=0$, then $u \equiv 0$ is optimal for all $x_{0}$ ). Now recently ([1]), a necessary and sufficient condition of solvability was given for the regular case in terms of the stabilizability of a suitable defined quotient system.

If the problem is singular, then it is known that optimal inputs need not exist within the class $C_{s m}^{m}$ ([7, Example 2.11]). With a reformulation in the style of [7] incorporating distributions as possible inputs, this extra difficulty can be dealt with and it is proven in [2] that the input class $C_{\mathrm{imp}}^{m}$ of impulsive-smooth distribution on $\mathbb{R}$ with support on $[0, \infty)([7$, Definition 3.1$])$ is large enough to be representative for the system's behaviour under general distributions as inputs. A distribution $u \in C_{\mathrm{imp}}^{m}$ can be written as a sum of a function $u_{2} \in C_{s m}^{m}$ and an impulsive distribution $u_{1}$ with support in $\{0\}$. Obviously, we require $u \in C_{\mathrm{imp}}^{\mathrm{m}}$ to be such that for every $x_{0}$ the resulting output $y\left(x_{0}, u\right)$ has no impulsive component, and the (system dependent) space of these inputs is denoted $U_{\Sigma}$. In [2, Proposition 4.5] an explicit description for this input class is given by means of a dual version of Silverman's structure algorithm. With the help of this generalized dual structure algorithm ([2, Section 4$])$, the necessary and sufficient condition for solvability of LQCP given in [1] can be generalized to singular problems ([1, Remark 5]).

In the present paper, it will be shown that the latter condition is equivalent to the condition $\mathbf{S}^{-}=\mathbb{R}^{n}$. In other words, output stabilizability is necessary and sufficient for solvability of LQCP. This intuitively rather obvious condition turns out to be relatively difficult to prove. 
In the sequel we will need the following well-known concepts. Let $V=V(\Sigma)=$ $\left\{x_{0} \in \mathbb{R}^{n} \mid \exists_{u \in C_{m}^{m}}: y\left(x_{0}, u\right) \equiv 0\right\}$ (the weakly unobservable subspace), then ([7, Theorem 3.10]) $\mathbf{V}$ is the largest subspace $\mathbf{L}$ for which there exists a feedback $F$ such that $(A+B F) \mathbf{L} \subset \mathbf{L}$, $(C+D F) \mathbf{L}=0$. Dually, $\mathbf{W}=\mathbf{W}(\Sigma)$ (the strongly reachable subspace) is the smallest subspace $\mathbf{K}$ for which there exists an "output injection" $G$ such that $(A+G C) \mathbf{K} \subset \mathbf{K}, \operatorname{im}(B+G D) \subset \mathbf{K}$ ([7, Theorem 3.15]) and $W \subset<A \mid \operatorname{im}(B)>$ (the reachable subspace). It is easily established that $\mathrm{W}=0$ if and only if $\operatorname{ker}(D)=0$.

Next, if $K \in \mathbb{R}^{n \times n}$ and $F(K):=\left[\begin{array}{cc}C^{\prime} C+A^{\prime} K+K A & K B+C^{\prime} D \\ B^{\prime} K+D^{\prime} C & D^{\prime} D\end{array}\right]$ (the dissipation matrix), then $K$ is said to satisfy the dissipation inequality if $K \in \Gamma:=\left\{K \in \mathbb{R}^{n \times n} \mid K=K^{\prime}, F(K) \geq 0\right\}$ ([9]). Note that $\Gamma \neq \varnothing(0 \in \Gamma)$. If $T(s):=D+C(s I-A)^{-1} B(s \in \mathcal{C})$ (the transfer function), and $\rho:=$ normal rank $(T(s))$, then it is proven in [8] that

\section{Lemma 1.1}

If $K \in \Gamma$, then rank $(F(K)) \geq \rho$.

Set $\Gamma_{\min }:=\{K \in \Gamma \mid \operatorname{rank}(F(K))=p\}$. This subset of $\Gamma$ is of importance because of the next result from [2].

\section{Proposition 1.2}

If $(A, B)$ is stabilizable, then there exists an element $K^{-} \in \Gamma_{\min } \cap\{K \in \Gamma \mid K \geq 0\}$ such that, for all $x_{0}, J^{-}\left(x_{0}\right)=x_{0} K^{-} x_{0}$.

$$
\begin{aligned}
& \text { If } \operatorname{ker}(D)=0 \text { and } \\
& \qquad \Phi(K):=C^{\prime} C+A^{\prime} K+K A-\left(K B+C^{\prime} D\right)\left(D^{\prime} D\right)^{-1}\left(B^{\prime} K+D^{\prime} C\right),
\end{aligned}
$$

then it is easily seen ([9]) that $\Gamma_{\min }=\left\{K \in \mathbb{R}^{n \times n} \mid K=K^{\prime}, \Phi(K)=0\right\}$, the set of solutions of the algebraic Riccati equation. Now a second major observation of this paper is, that $\Gamma_{\min } \cap\{K \in \Gamma \mid K \geq 0\} \neq \varnothing$ if and only if $S^{-}=\mathbb{R}^{n}$. Hence, in the regular case, there exists a positive semi-definite solution of the algebraic Riccati equation if and only if $\Sigma$ is output stabilizable.

\section{The dual structure algorithm and the output-stabilizable subspace}

If $q_{0}:=\operatorname{rank}(D)$, then there exists a regular transformation $S_{0}$ such that $D S_{0}=\left[D_{0}, 0\right]$ with $D_{0}$ left invertible and we will take $S_{0}=I_{m}$ if $q_{0}=m$ (note that $S_{0}$ can be chosen such that 
$\left.S_{0}^{-1}=S_{0}{ }^{\prime}\right)$. Set $B S_{0}=:\left[\bar{B}_{0}, \tilde{B}_{0}\right]$, then substitution of $u=S_{0}\left[\bar{w}_{0}{ }^{\prime}, \tilde{w}_{0}{ }^{\prime}\right]^{\prime}$ into (1.1) yields

$$
\dot{x}=A x+\bar{B}_{0} \bar{w}_{0}+\tilde{B}_{0} \tilde{w}_{0}, x_{0}, y=C x+D_{0} \bar{w}_{0},
$$

and $\tilde{B}_{0}$ is left invertible, $\operatorname{im}\left(\tilde{B}_{0}\right) \subset \mathrm{W}$. This input transformation corresponds to the first part of step 0 of the generalized dual structure algorithm ([2, Section 4$])$. Notice that $\tilde{B}_{0}$ is not appearing if $q_{0}=m$. In fact, the dual algorithm is a void concept if $\operatorname{ker}(D)=0$. If $\operatorname{ker}(D) \neq 0$, then this algorithm transforms the given system $\Sigma$ into a system $\Sigma_{\alpha}(\alpha$ an integer, not less than 1$)$ of the form

$$
\begin{aligned}
& \dot{x}_{\alpha}=A x_{\alpha}+\underline{\bar{B}} \bar{w}_{\alpha}+\hat{B} \hat{w}, x_{0}, \\
& y=C x_{\alpha}+\underline{D} \bar{w}_{\alpha},
\end{aligned}
$$

where $\underline{B}=\left[\bar{B}_{0}, \bar{B}_{\text {add }}\right], \underline{D}=\left[D_{0}, D_{\text {add }}\right], \bar{B}_{\text {add }}$ is an $n \times\left(\rho-q_{0}\right)$ real matrix which is such that $\operatorname{im}\left(\bar{B}_{\text {add }}\right) \subset A(\mathbf{W}), D_{\text {add }}$ is a $r \times\left(p-q_{0}\right)$ real left invertible matrix, and $\operatorname{rank}(\underline{D})=\rho$, $C(\mathbf{W}) \subset \operatorname{im}(\underline{D})$ and $\operatorname{im}(\hat{B}) \subset \mathbf{W}$. Moreover, the control $u \in C_{\mathrm{imp}}^{m}$ and the input $\left[\bar{w}_{\alpha}{ }^{\prime} \hat{w}^{\prime}\right]^{\prime}$ are linked by $u=H(p)\left[\bar{w}_{\alpha}^{\prime} \hat{w}^{\prime}\right]^{\prime}$, where $H(s)$ is an invertible polynomial matrix, $p$ stands for the derivative of Diracs $\delta$ distribution and $H(p)$ thus is the matrix-valued distribution obtained by substituting $s=p$ into $H(s)$. Finally, for all $t>0$, we have that $\left(x\left(x_{0}, u\right)(t)-\right.$ $\left.x_{\alpha}\left(x_{0},\left[\bar{w}_{\alpha}^{\prime}, \hat{w}^{\prime}\right]^{\prime}\right)(t)\right) \in \mathbf{W}$. Now, let us apply to $(2.2)$ the preliminary state feedback law

$$
\bar{w}_{\alpha}=-\left(\underline{D}^{\prime} \underline{D}\right)^{-1} \underline{D}^{\prime} C x_{\alpha}+\hat{w}_{\alpha} .
$$

Then we get

$$
\dot{x}_{\alpha}=\underline{A} x_{\alpha}+\underline{B} \hat{w}_{\alpha}+\hat{B} \hat{w}, x_{0}, y=\underline{C} x_{\alpha}+\underline{D} \hat{w}_{\alpha}
$$

with $\underline{A}:=A-\underline{B}\left(\underline{D}^{\prime} \underline{D}\right)^{-1} \underline{D}^{\prime} C, \underline{C}:=\left(I_{r}-\underline{D}\left(\underline{D^{\prime}} \underline{D}\right)^{-1} \underline{D^{\prime}}\right) C$.

From $[2$, Lemmas $4.2-4.4]$ and the above we then have the following.

\section{Proposition 2.1}
a) $\quad \underline{W} \subset \mathbf{W}$.
b) $\quad \mathrm{V}\left(\Sigma_{\mathrm{\alpha}}\right)=\mathrm{V}(\Sigma)+\mathrm{W}(\Sigma)=\langle\operatorname{ker}(\underline{C}) \mid \underline{A}\rangle$.
c) $\langle A \mid \operatorname{im}(\bar{B}, \hat{B})\rangle+\mathrm{W}=\langle A \mid \operatorname{im}(B)\rangle$.

One consequence of Proposition 2.1 is, that $y$ is independent of $\hat{w}$; we may just as well take $\hat{w}=0$. Now let us define (where $y(\infty)$ denotes $\lim _{t \rightarrow \infty} y\left(x_{0}, u\right)(t)$ )

$$
\mathbf{T}_{1}:=\left\{x_{0} \in \mathbb{R}^{n} \mid \exists_{u \in U_{x}}: y(\infty)=0\right\}
$$


and $\mathrm{T}_{2}:=\left\{x_{0} \in \mathbb{R}^{n} \mid \exists_{u \in U_{\Sigma}}: J\left(x_{0}, u\right)<\infty\right\}$,

then we establish that $\mathbf{T}_{1}=\left\{x_{0} \mid \exists_{\hat{w}_{\alpha}, \text { smooth }}:\left(\underline{C} x_{\alpha}+\underline{\underline{D}} \hat{w}_{\alpha}\right)(\infty)=0\right\}$ and $\mathbf{T}_{2}=\left\{x_{0} \mid \exists_{\hat{w}_{\alpha} \text { smooth }}\right.$ : $\left.\int_{0}^{\infty}\left[\underline{C} x_{\alpha}+\underline{D} \hat{w}_{\alpha}\right]^{\prime}\left[\underline{C} x_{\alpha}+\underline{D} \hat{w}_{\alpha}\right] d t<\infty\right\}$ with $x_{\alpha}(t)=\exp (A t) x_{0}+\int_{0}^{t} \exp (A(t-\tau)) \underline{B} \hat{w}_{\alpha}(\tau) d \tau$ and hence $\mathbf{T}_{1,2}$ are $\Sigma_{\alpha}$-invariant ([5, Def. 2.2]).

Next, let

$$
\mathrm{S}^{-}(\Sigma):=X^{-}(A)+<A \mid \operatorname{im}(B)>+\mathrm{V}(\Sigma)
$$

(where $X^{-}(A)$ denotes the stable subspace of $A$ ). Then it is rather obvious that $\mathbf{S}^{-}(\Sigma) \subset \mathbf{T}_{i}(i=1,2)$ and that (Proposition 2.1) $\mathbf{S}^{-}\left(\Sigma_{\alpha}\right)=\mathbf{S}^{-}(\Sigma)=: \mathbf{S}^{-}$. Therefore ([5, Remark 2.26]) $\mathbf{T}_{1,2}$ are strongly $\Sigma_{\alpha}$-invariant and we thus have found that $\mathbf{V}\left(\Sigma_{\alpha}\right) \subset \mathbf{S}^{-} \subset \mathbf{T}_{i}$ and $\underline{A} \mathbf{V}\left(\Sigma_{\alpha}\right)$ $\subset \mathbf{V}\left(\Sigma_{\alpha}\right), \underline{A} \mathbf{S}^{-} \subset \mathbf{S}^{-}, \underline{A} \mathbf{T}_{i} \subset \mathbf{T}_{i}(i=1,2)$. Let $X_{2}, X_{3}, X_{4}$ be such that $\mathbf{V}\left(\Sigma_{\alpha}\right) \oplus X_{2}=\mathbf{S}^{-}$, $\mathrm{S}^{-} \oplus X_{3}=\mathrm{T}_{1}, \mathrm{~T}_{1} \oplus X_{4}=\mathbb{R}^{n}$. By choosing appropriate basis matrices for these subspaces, (2.4a) (with $\hat{w}=0)$ transforms into

$$
\begin{aligned}
& {\left[\begin{array}{c}
\dot{x}_{1} \\
\dot{x}_{2} \\
\dot{x}_{3} \\
\dot{x}_{4}
\end{array}\right]=\left[\begin{array}{cccc}
A_{11} & A_{12} & A_{13} & A_{14} \\
0 & A_{22} & A_{23} & A_{24} \\
0 & 0 & A_{33} & A_{34} \\
0 & 0 & 0 & A_{44}
\end{array}\right]\left[\begin{array}{c}
x_{1} \\
x_{2} \\
x_{3} \\
x_{4}
\end{array}\right]+\left[\begin{array}{c}
B_{1} \\
B_{2} \\
0 \\
0
\end{array}\right] \hat{w}_{\alpha},\left[\begin{array}{c}
x_{01} \\
x_{02} \\
x_{03} \\
x_{04}
\end{array}\right],} \\
& y=\left[\begin{array}{llll}
0 & C_{2} & C_{3} C_{4}
\end{array}\right]\left[\begin{array}{l}
x_{1} \\
x_{2} \\
x_{3} \\
x_{4}
\end{array}\right]+\underline{D} \hat{w}_{\alpha},\left[\left[C_{2} C_{3} C_{4}\right],\left[\begin{array}{ccc}
A_{22} & A_{23} & A_{24} \\
0 & A_{33} & A_{34} \\
0 & 0 & A_{44}
\end{array}\right]\right] \text { is observable . }
\end{aligned}
$$

Note that $\sigma\left(A_{33}\right) \cup \sigma\left(A_{44}\right) \subset \overline{\boldsymbol{C}}^{+}$(since $X^{-}(A) \subset \mathbf{S}^{-}\left(\Sigma_{\alpha}\right)$ ). Now take a point $x_{0} \in \mathbf{T}_{1}$, i.e. $x_{04}=0$ in (2.7) (and thus $x_{4} \equiv 0$ ). Since $\underline{D^{\prime}} \underline{C}=0$ and $\underline{D}$ is left invertible, it follows that $\left(C_{2} x_{2}+C_{3} x_{3}\right)(\infty)=0, \hat{w}_{\alpha}(\infty)=0$, and thus ([3, Chapter 3]) that $x_{2}(\infty)=0, x_{3}(\infty)=0$ (i.e., $x\left(x_{0}, u\right)(t)$ converges to $\left.\mathrm{V}+\mathrm{W}(t \rightarrow \infty)\right)$. Hence, necessarily, $x_{03}=0$ and we establish that $\mathbf{T}_{1}=\mathbf{S}^{-}$. In the same way we find that $\mathbf{T}_{2}=\mathbf{S}^{-}$. If for every $F \in \mathbb{R}^{m \times n}$, we define the spaces

$$
\begin{aligned}
& \mathbf{T}_{1}^{F}:=\left\{x_{0} \in \mathbb{R}^{n} \mid \text { if } u=F x \text {, then } y\left(x_{0}, u\right)(\infty)=0\right\}, \\
& \mathbf{T}_{2}^{F}:=\left\{x_{0} \in \mathbb{R}^{n} \mid \text { if } u=F x \text {, then } \int_{0}^{\infty} y^{\prime}\left(x_{0}, u\right) y\left(x_{0}, u\right) d t<\infty\right\},
\end{aligned}
$$

we thus have arrived at our first main result. 


\section{Theorem 2.2}

Consider the system $\Sigma$ and the corresponding subspaces defined above. Then $\mathbf{T}_{i}=\mathbf{S}^{-}$and $\mathbf{T}_{i}^{F} \subset \mathbf{S}^{-}$for every $F \in \mathbb{R}^{m \times n}$. In addition, there exists an $F \in \mathbb{R}^{m \times n}$ such that $\mathbf{T}_{i}^{F}=\mathbf{S}^{-}(i=1,2)$.

Proof. Let $F \in \mathbb{R}^{m \times n}$ be given. If we use the feedback $u=F x$, then the resulting output $y$ will tend to zero exponentially fast when either $x_{0} \in \mathbf{T}_{1}^{F}$ or $x_{0} \in \mathbf{T}_{2}^{F}$ and thus $\mathbf{T}_{1}^{F}=\mathbf{T}_{2}^{F}$. In addition, it is trivial that $\mathbf{T}_{1}^{F}=\mathbf{T}_{2}^{F} \subset \mathbf{T}_{i}(i=1,2)$. The fact that there exists an $F$ such that $\mathbf{T}_{1}^{F}=\mathbf{S}^{-}$is known (compare [5]). The rest follows from the above.

Because of the relation $\mathbf{T}_{1}^{F}=\mathbf{S}^{-}$for some $F$, we will refer to $\mathbf{S}^{-}$as the output-stabilizable subspace.

\section{The dual structure algorithm and optimal control}

Let us reconsider the LQCP and assume that $\mathbf{S}^{-}=\mathbb{R}^{n}$. According to Theorem 2.2, we can reformulate this as: For every $x_{0}$ there exists an input $u \in U_{\Sigma}$ such that $J\left(x_{0}, u\right)<\infty$. Clearly this is a necessary condition for the solvability of LQCP. Since $y=C x_{\alpha}+D \hat{w}_{\alpha}$ with $\underline{D}$ left invertible, we are left with a regular LQCP by taking $\hat{w}=0$ in (2.4a). Hence we may apply the second part of the proof of the main Theorem in [1] and state that the algebraic Riccati equation associated with (2.4a), $\tilde{\Phi}(K)=0$ with

$$
\tilde{\Phi}(K):=\underline{C}^{\prime} \underline{C}+\underline{A}^{\prime} K+K \underline{A}-K \underline{\bar{B}}\left(\underline{D^{\prime}} \underline{D}\right)^{-1} \underline{B}^{\prime} K,
$$

has a solution $K^{-} \geq 0$ and that every other solution $K \geq 0$ of $\tilde{\Phi}(K)=0$ satisfies $K \geq K^{-}$. The optimal cost for LQCP, $J^{-}\left(x_{0}\right)$, equals $x_{0}{ }^{\prime} K^{-} x_{0}$ for all $x_{0}, \operatorname{ker}\left(K^{-}\right)=\mathrm{V}+\mathbf{W}$ and, in addition, for every $x_{0}$ an optimal control for LQCP exists (see for details [2, Theorem 4.5]) and thus the condition $S^{-}=\mathbb{R}^{n}$ is also sufficient for solvability of LQCP. Now in [2, Section 6] the next result is proven.

\section{Proposition 3.1}

$$
\begin{aligned}
& \Gamma=\left\{K \in K^{\prime}, \mathrm{W} \subset \operatorname{ker}(K), \tilde{\Phi}(K) \geq 0\right\}, \\
& \Gamma_{\min }=\left\{K \in \mathbb{R}^{n \times n} \mid K=K^{\prime}, \mathrm{W} \subset \operatorname{ker}(K), \tilde{\Phi}(K)=0\right\} .
\end{aligned}
$$

Consequently, we observe that $K^{-} \in \Gamma_{\min } \cap\{K \in \Gamma \mid K \geq 0\}$ and every other $K \in \Gamma_{\min } \cap$ $\{K \in \Gamma \mid K \geq 0\}$ satisfies $K \geq K^{-}$(compare Proposition 1.2). Note that $\tilde{\Phi}(K)=\Phi(K)$ if $\operatorname{ker}(D)=0$. Therefore, in the regular case, $K^{-}$represents the smallest positive semi-definite solution of (1.3). On the other hand, if $\Gamma_{\min } \cap\{K \in \Gamma \mid K \geq 0\} \neq \varnothing$, then ([3, Chapter 3]) $\mathbf{S}^{-}=\mathbb{R}^{n}$. Hence 


\section{Theorem 3.2}

$S^{-}=\mathbb{R}^{n}$ if and only if $\Gamma_{\min } \cap\{K \in \Gamma \mid K \geq 0\} \neq \varnothing$. In addition, if the latter set is nonempty, then the smallest element of this set, $K^{-}$, represents the optimal cost for the LQCP.

Note that the characterization of $K^{-}$as given above is formulated directly in terms of the original system data $(A, B, C, D)$. Moreover, this representation of the optimal cost includes the singular as well as the regular case. Finally, we mention that a condition for output stabilizability can be given in the spirit of [4]. In fact, a more general formulation is

\section{Proposition 3.3 ([3, Chapter 3])}

Let $\mathbf{T}$ be a $\Sigma$-invariant subspace. Then $X^{-}(A)+\langle A| \operatorname{im}(B)>+\mathbf{T}=\mathbb{R}^{n}$ if and only if $\forall_{\lambda \in \bar{C}^{+}}$ $\forall \forall_{\eta \in C}:\left[\eta\left(A-\lambda_{n}, B\right)=0\right.$ and $\left.\eta \mathrm{T}=0\right] \Rightarrow \eta=0$.

The condition for output stabilizability is obtained by taking $T=V$.

\section{Remarks}

1. While proving out main Theorem 2.2, we established that if $u \in U_{\Sigma}$ is such that $y\left(x_{0}, u\right)(\infty)=0$ or $J\left(x_{0}, u\right)<\infty$, then $x\left(x_{0}, u\right)(t)$ converges to $\mathrm{V}+\mathrm{W}(t \rightarrow \infty)$, but not necessarily to $\mathbf{V}$ (for a counterexample, see [6]), unless (of course) $\mathbf{W}=0$, i.e. $\operatorname{ker}(D)=0$.

2. Since $\mathbf{S}^{-} \subset \tilde{\mathbf{T}}_{1}:=\left\{x_{0} \mid \exists_{u \in C_{s m}^{m}}: y(\infty)=0\right\} \subset \mathbf{T}_{1}=\mathbf{S}^{-}$, we find that $\tilde{\mathbf{T}}_{1}=\mathbf{T}_{1}$, and, analogously, that $\tilde{\mathbf{T}}_{2}:=\left\{x_{0} \mid \exists_{u \in C_{m}^{n}}: J\left(x_{0}, u\right)<\infty\right\}=\mathbf{T}_{2}$. In fact, this can be seen directly as $\mathbf{T}_{i}=$ $\mathbf{W}+\tilde{\mathbf{T}}_{i}=\tilde{\mathbf{T}}_{i}$ because $\mathbf{W} \subset<A \mid \operatorname{im}(B)>\subset \tilde{\mathbf{T}}_{i}(i=1,2)$.

3. If $\mathbb{R}^{n}:=\mathbb{R}^{n} /(\mathrm{V}+\mathrm{W}), \bar{A}: \overline{\mathbb{R}}^{n} \rightarrow \overline{\mathbb{R}}^{n}$ denotes the induced map of $\underline{A}$ defined by $\bar{A} \bar{x}:=$ $(\underline{\bar{A}} x)(\bar{x}=x+(\mathbf{V}+\mathbf{W}))$ and $\underline{\bar{B}}: \mathbb{R}^{m} \rightarrow \overline{\mathbb{R}}^{n}$ is defined by $\underline{\bar{B}} u:=(\underline{\bar{B}} u)$, then it can be seen (e.g. compare $[2$, Lemma 5.6]) that the condition in Proposition 3.3 with $\mathbf{T}=\mathbf{V}$ is equivalent to: $(\underline{\bar{A}}, \underline{\bar{B}})$ is stabilizable. Hence, in accordance with [1, Remark 5], the latter condition is necessary and sufficient for the solvability of LQCP.

\section{Acknowledgement}

This research was supported by the Netherlands organization for scientific research (N.W.O.). 


\section{References}

[1] Ton Geerts, "A necessary and sufficient condition for solvability of the linear-quadratic control problem without stability", Syst. \& Contr. Lett., vol. 11, pp. 47-51, 1988.

[2] Ton Geerts, "All optimal controls for the singular linear-quadratic problem without stability; a new interpretation of the optimal cost", Lin. Alg. \& Appl., vol. 116, pp. 135-181, 1989.

[3] Ton Geerts, PhD Thesis, forthcoming.

[4] M.L.J. Hautus, "Stabilization, controllability and observability of linear autonomous systems", Nederl. Akad. Wetensch. Proc. Ser. A, 73, pp. 448-455, 1970.

[5] M.L.J. Hautus, " $(A, B)$-invariant and stabilizability subspaces, a frequency domain description", Automatica, vol. 16, pp. 703-707, 1980.

[6] M.L.J. Hautus, "Strong detectability and observers", Lin. Alg. \& Appl., vol. 50, pp. 353-368, 1983.

[7] M.L.J. Hautus \& L.M. Silverman, "System structure and singular control", Lin. Alg. \& Appl., vol, 50, pp. 369-402, 1983.

[8] J.M. Schumacher, "The role of the dissipation matrix in singular optimal control", Syst. \& Contr. Lett., vol. 2, pp. 262-266, 1983.

[9] J.C. Willems, "Least squares stationary optimal control and the algebraic Riccati equation", IEEE Trans. Automat. Contr., vol. AC-16, pp. 621-634, 1971.

[10] J.C. Willems, A. Kitapçi \& L.M. Silverman, "Singular optimal control: A geometric approach", SIAM J. Contr. \& Opt., vol. 24, pp. 323-337, 1986.

[11] W.M. Wonham, Linear Multivariable Control: A Geometric Approach, Springer, New York, 1979. 